*Correspondencia:

gabbyta280587@gmail.com

Ave 12 de Octubre 1076, Quito Código postal 170143. Teléfono: 593 (02) 2991604

Conflicto de intereses: Los autores declaran no tener conflictos de intereses.

Recibido: 14 de Mayo 2021

Aceptado: 25 de Octubre, 2021

Publicado: 9 de Diciembre, 2021

Editor: Dr. Felipe Campoverde

\section{Membrete bibliográfico:} Ordoñez G, Navas V, Buitrón R. Estudio actuarial de supervivencia en pacientes con cáncer colorrectal, atendidos en la unidad de oncología del Hospital Eugenio Espejo, Quito, periodo 2010 - 2016. Rev. Oncol. Ecu 2021;31(3):201-212.

ISSN: 2661-6653

DOI: https://doi.org/10.33821/589

Copyright Ordóñez G, et al. Este artículo es distribuido bajo los términos de Creative Commons Attribution License BY-NCSA 4.0, el cual permite el uso y redistribución citando la fuente y al autor original.
Estudio actuarial de supervivencia en pacientes con cáncer colorrectal, atendidos en la unidad de oncología del Hospital Eugenio Espejo, Quito, periodo 2010 - 2016

\section{Actuarial survival study in patients with colorectal cancer, treated in the oncology unit of Hospital Eu- genio Espejo, Quito, period 2010 - 2016}

\section{María Gabriela Ordóñez Ureta*1i, Rafael Vinicio Navas Flores ${ }^{2}$, Rene Buitrón Andrade1 1 (D)}

1. Facultad de Ciencias Médicas, Pontificia Universidad Católica del Ecuador, QuitoEcuador.

2. Servicio de Oncología, Hospital de Especialidades Eugenio Espejo, Ministerio de Salud Pública, Quito-Ecuador

Resumen

Introducción: La supervivencia relativa a los cinco años para pacientes con cáncer colorrectal (CCR) se presenta entre el $60 \%$ en América del Norte y de $40 \%$ o menos en Argelia y Estonia. Es objetivo del presente estudio establecer la supervivencia a nivel local y determinar los factores asociados en un estudio de un centro nacional de referencia en Quito, Ecuador.

Metodología: El presente estudio observacional se realizó en el Hospital de Especialidades Eugenio Espejo de la ciudad de Quito-Ecuador, en el período de enero del 2010 a diciembre del 2016. Se incluyeron pacientes con cáncer colorrectal. Las variables fueron descriptivas demográficas, variables clínicas y mortalidad. La muestra fue no probabilística, tipo censo. La variable mortalidad, fue estudiada con las variables Independientes clínicas, epidemiológicas con análisis actuarial de supervivencia.

Resultados: Se incluyeron 395 casos, el 62.3\% mujeres, con una edad 60 años (16 a 94). El 7.1\% de casos refirieron algún tipo de exposición de riesgo relacionado a CCR. La supervivencia global fue del $56 \%$, con una tasa de mortalidad del 7\%. Casos con CCR estadio I sobrevida a los 6 años de 100\%, con estadio II $96.5 \%$, en el estadio III el 84\%, estadio IV de 63\% ( $P=0.001)$. Pacientes con instrucción secundaria o superior con supervivencia del $95 \%$ versus $88 \%$ en pacientes con instrucción primaria/analfabetos ( $P=0.048)$. La supervivencia es mayor en pacientes con diagnóstico temprano estadios (I y II) ( $P=0.007)$. 
Conclusión: La supervivencia en el presente estudio se asoció al nivel de instrucción de los pacientes, al estadiaje del cáncer, y al diagnóstico temprano sobre todo en pacientes con comorbilidades. No se evidenció relación con factores de riesgo como tabaco, alcohol, presencia de pólipos, etc.

\section{Palabras claves:}

DeCS: Neoplasias del Colon, Neoplasias Colorrectales, Factores de Riesgo, Estilo de Vida, Análisis de Supervivencia, Detección Precoz del Cáncer.

DOI: $10.33821 / 589$

\section{Abstract}

Introduction: The five-year relative survival for patients with colorectal cancer (CRC) is between 60\% in North America and 40\% or less in Algeria and Estonia. The objective of this study is to establish survival at the local level and determine the associated factors in a study of a national referral center in Quito, Ecuador.

Methodology: The present observational study was carried out at the Eugenio Espejo Specialty Hospital in the city of Quito-Ecuador, in the period from January 2010 to December 2016. Patients with colorectal cancer were included. The variables were descriptive demographic, clinical variables and mortality. The sample was non-probabilistic, census type. The mortality variable was studied with the independent clinical and epidemiological variables with actuarial survival analysis.

Results: A total of 395 patients were analyzed, 62.3\% women, aged 60 years (16 to 94). 7.1\% of cases referred to some type of risk exposure related to CRC. Overall survival was $56 \%$, with a mortality rate of $7 \%$. Cases with stage I CRC survival at 6 years of $100 \%$, with stage II $96.5 \%$, in stage III $84 \%$, stage IV of $63 \%(P=0.001)$. Patients with secondary education or higher with survival of $95 \%$ versus $88 \%$ in patients with primary education / illiterate $(P=0.048)$. Survival is higher in patients with early diagnosis stages (I and II) $(P=0.007)$.

Conclusion: Survival in the present study was associated with the level of education of the patients, the staging of the cancer, and early diagnosis, especially in patients with comorbidities. There was no evidence of a relationship with risk factors such as tobacco, alcohol, presence of polyps, etc.

\section{Keywords:}

MESH: Colonic Neoplasms, Colorectal Neoplasms, Risk Factors, Life Style, Survival Analysis, Early Detection of Cancer.

\section{Introducción}

Los resultados del estudio Concord muestran que la supervivencia relativa (SR) a los cinco años para el cáncer colorrectal (CCR) durante el periodo 1990-1999 osciló entre 60\% en América del Norte, Japón, Australia y algunos países de Europa occidental, hasta 40\% o menos en Argelia, República Checa, Estonia, Polonia, Eslovenia y Gales. La estimación de la SR en Cuba 
fue de $60 \%$ en hombres mujeres. En el resto de países de Latinoamérica es muy limitada la información disponible [1].

En Ecuador se registran aproximadamente 1200 casos anualmente según cifras oficiales de la Sociedad de Lucha Contra el Cáncer (SOLCA), de estos casos, si son tratados oportunamente, el $52 \%$ de personas sobreviven [2].

La supervivencia es una técnica de análisis de datos, utilizada para calcular el tiempo transcurrido desde el inicio del seguimiento de un evento, hasta el momento en que este se presenta si es que lo hace, en el plazo de un tiempo previamente determinado de estudio.9 Se estima que hay 14 millones de sobrevivientes de cáncer en Estados Unidos; y, cerca de 30 millones en el mundo [즌 $]$. El seguimiento del paciente con cáncer se lo hace en el periodo de mayor riesgo de recurrencia, por lo general 5 años [3].

El cáncer colorrectal (CCR) es una entidad patológica que afecta significativamente a la humanidad, a pesar del desarrollo tecnológico, la implementación de nuevas terapias y el avance farmacológico presenta una alta mortalidad. A nivel mundial el cáncer colorrectal es la tercera causa de muerte por cáncer en ambos sexos, pero en países desarrollados llega a ser la segunda. Las tasas de supervivencia a cinco años son significativamente diferentes por estadio, siendo de $90 \%$ para enfermedad localizada y de 10\% para enfermedad a distancia, evidentemente ligada a la detección temprana. En función a la detección temprana existen notables avances en el tamizaje, diagnóstico y tratamiento [4].

La mortalidad por CCR ha disminuido progresivamente desde mediados de 1980 en Estados Unidos y países occidentales, lo cual ha aumentado el número de sobrevivientes. Se espera que estos sobrevivientes tengan los efectos normales del envejecimiento, quizá agravados por haber tenido cáncer [5].

El objetivo del presente estudio fue determinar la supervivencia de los pacientes con CCR tratados en el Servicio de Oncología del Hospital de Especialidades Eugenio Espejo, QuitoEcuador entre los años 2010-2016, y sus factores asociados.

\section{Materiales y métodos \\ Diseño del estudio}

El estudio corresponde a un estudio de tipo observacional de supervivencia.

\section{Escenario}

El estudio de se realizó en el servicio de estadística y de oncología clínica del Hospital de Especialidades Eugenio Espejo de la ciudad de Quito-Ecuador. El período del estudio es 1 de enero del 2010 al 31 de diciembre del 2016. El período de campo terminó el 20 de enero del 2017. El informe se terminó el 25 de abril del 2017.

\section{Participantes}

Se incluyeron pacientes diagnosticados y tratados de cáncer colorrectal en el Servicio de Oncología del Hospital de Especialidades Eugenio Espejo en el periodo establecido, también se incluyeron pacientes derivados desde otras Unidades de Salud con diagnóstico de cáncer colorrectal que iniciaron protocolo de tratamiento. Se excluyeron pacientes con menos de 30 días de diagnóstico en el Hospital Eugenio Espejo y pacientes con neoplasia colorrectal se- 
cundario a metástasis de carcinoma procedente de otro órgano. Para el tamizaje en el departamento de estadística se utilizaron los siguientes códigos de la clasificación internacional de enfermedades 10ma edición: C18 Neoplasia maligna de colon, C18.0 Neoplasia maligna de ciego, C18.1 Neoplasia maligna de apéndice, C18.2 Neoplasia maligna de colon ascendente, C18.3 Neoplasia maligna de ángulo hepático, C18.4 Neoplasia maligna de colon transverso, C18.5 Neoplasia maligna de ángulo esplénico, C18.6 Neoplasia maligna de colon descendente, izquierdo (L), C18.7 Neoplasia maligna de colon sigmoide, C18.8 Neoplasia maligna de localizaciones contiguas de colon, C18.9 Neoplasia maligna de colon, no especificada, C19 Neoplasia maligna de unión rectosigmoidea, C20 Neoplasia maligna de recto.

\section{Variables}

Las variables fueron descriptivas demográficas: sexo, edad, estado civil, nivel de educación, ocupación. Variables clínicas: estado nutricional, pruebas diagnósticas y mortalidad.

\section{Fuentes de datos / medición}

Para cada variable se utilizó el software institucional para registro de historias clínicas como fuente de datos, se consultó el expediente clínico electrónico, adicionalmente se consultó el expediente físico. Los datos fueron compilados en una hoja electrónica para posteriormente ser transferidos al software estadístico.

\section{Control de las fuentes de sesgo.}

Se excluyeron historias clínicas cuyos datos no estuvieron completos, se evitó la imputación de datos perdidos o excluidos. Se realizó un doble chequeo de los datos por fuentes independientes, de los servicios de hospitalización y de los registros en el área de estadística.

\section{Tamaño del estudio}

La muestra fue no probabilística, tipo censo en la cual se incluyeron todos los casos potencialmente elegibles de la institución del período de estudio (7 años).

\section{Manejo de variables cuantitativas}

Las variables cuantitativas en escala se presentan con promedios y desviación estándar. Las variables cuantitativas nominales se presentan con frecuencia y porcentaje.

\section{Métodos Estadísticos}

La variable dependiente fue la mortalidad, la cual fue estudiada con las variables Independientes clínicas, epidemiológicas con análisis actuarial de supervivencia El paquete estadístico utilizado fue SPSS v.23 para Windows (Armonk, NY: IBM Corp).

\section{Resultados}

En el estudio se incluyeron 395 casos.

\section{Características demográficas}

Se analizó un total de 395 pacientes diagnosticados de cáncer colon en el Servicio de Oncología del Hospital de Especialidades Eugenio Espejo, en el periodo 2010 - 2016, de los cuales 
el 62.3\% pertenecen al género femenino y el $37.7 \%$ al género masculino. La edad promedio entre los pacientes en estudio fue de 60 años, con una edad mínima al momento del diagnóstico de 16 años y la máxima de 94 años y una moda de 65 años.

El $6.1 \%$ de los pacientes viven en unión libre, $51.6 \%$ son casados, el $17.2 \%$ solteros, $15.7 \%$ divorciados y $9.4 \%$ viudos. El $62.3 \%$ corresponden al nivel de educación primaria, el $27.1 \%$ terminó la secundaria y el 5.8\% fueron de instrucción superior. Según su lugar de residencia habitual, el $91.4 \%$ vive en la región sierra, el 6.8\% en la región costa y en la región amazónica vive el 1.8\%. Las principales ocupaciones de los pacientes fueron: quehaceres domésticos $50.1 \%$, comerciante $13.7 \%$, agricultor $7.8 \%$, jubilado (sin especificar ocupación) 5.3\%, chofer $5.1 \%$, jornalero $3 \%$. La mortalidad fue del $7 \%$.

\section{Condición nutricional de los pacientes}

El $60.8 \%$ tuvieron normo peso, el $24.8 \%$ sobrepeso, mientras que el $7.7 \%$ obesidad. El $6.8 \%$ de los pacientes se encontraba en el momento del estudio en desnutrición hasta estados de caquexia.

\section{Pruebas diagnósticas}

En la evaluación diagnostica tacto rectal el momento de la colonoscopia se realizó al 90.6\% de los pacientes, sangre oculta en heces o prueba de guayaco se realizó en el $14.5 \%$ de los pacientes, con resultados positivos en el 5.1\%; la rectosigmoideoscopia se realizó en el 12.4\% de los pacientes con diagnóstico confirmado; la ecosonografía se realizó en el 35.7\% de los pacientes y la tomografía axial computarizada se practicó en el 95.4\%., el 75.2\% no reporta valores de laboratorio de proteínas en sangre, tampoco hay información en historias clínicas acerca de tipo de dieta o alimentación

Por último, se realizaron pruebas de marcadores tumorales para evaluar la respuesta del paciente al tratamiento o para monitorear si el cáncer recidiva después del tratamiento: El antígeno carbohidrato 19-9 (CA 19-9) se reporta en el 75,7\% de los pacientes en estudio, el $80 \%$ tiene valores por encima de $33 \mathrm{u} / \mathrm{ml}$ siendo este el valor de referencia máximo en laboratorio, los valores máximos de los pacientes llegan a elevarse hasta $3000 \mathrm{u} / \mathrm{ml}$ aproximadamente (28).

\section{Análisis de supervivencia}

\section{Supervivencia global de cáncer de colon}

La Supervivencia Global de los pacientes con diagnóstico de cáncer de colon en el Hospital Eugenio Espejo fue de 56\% en el periodo 2010-2016.

\section{Antecedentes familiares}

La supervivencia en los pacientes con antecedentes familiares de cáncer a 6 años fue del $97 \%$ y de quienes no tienen antecedentes familiares del $45 \%$. ( $P=0.543)$.

\section{Género}

En este estudio la supervivencia del género femenino en 6 años es del 59\% y la del género masculino es del $50 \%$. $(P=0.146)$. 


\section{Edad}

Se procedió a agrupar la edad de los pacientes según las etapas del ciclo vital observándose en el periodo estudiado, la supervivencia del cáncer colorrectal en la adolescencia es del 100\% de los pacientes, mientras que en etapas posteriores como la adultez media el $79 \%$ de los pacientes sobreviven; en la adultez posterior la supervivencia es del $17 \%$, y en la senectud el 91\% $(P=0.971)$.

\section{Estado civil}

Durante todo el periodo estudiado se observó una menor supervivencia en pacientes solteros, seguido de viven en unión libre, casados, y divorciados ( $P=0.694)$.

\section{Nivel de instrucción}

Los pacientes con instrucción superior y secundaria presentaron una supervivencia del 95\% en el periodo estudiado, la misma que fue menor (88\%) en los pacientes instrucción primaria y analfabetos $(P=0.048)$.

\section{Región de residencia habitual}

En la región costa la supervivencia fue del 59\%, en la sierra del $57 \%$ y en la región amazónica del $100 \%$ de los pacientes $(P=0.793)$.

\section{Consumo de alcohol}

El 21.6\% de los pacientes indican haber consumido alcohol, sin especificar la frecuencia, de ellos, el $74 \%$ de sobrevive durante todo el periodo estudiado en tanto que la supervivencia en el grupo que no lo consume fue del 38\% ( $P=0.529)$.

\section{Consumo de tabaco}

El 25.6\% reportaron consumir tabaco, sin especificar la frecuencia o cantidad, observándose que la supervivencia a 6 años de los pacientes que afirman haberlo consumido fue del 33\%; mientras en quienes no lo consumieron fue del $86 \%(P=0.286)$.

\section{Antecedentes familiares}

El 97\% de los pacientes con antecedentes de cáncer en la familia sobrevive en el periodo estudiado desde el diagnóstico, con una supervivencia del $45 \%$ en pacientes sin antecedentes de cáncer familiar ( $P=0.543)$.

\section{Sangre oculta en heces}

En los 6 años de estudio, el 100\% de los pacientes a quienes se les hizo el examen de sangre oculta en heces sobrevivieron, porcentaje que se redujo al $78 \%$ de quienes tuvieron resultados negativos en este examen, en tanto que el $55 \%$ de quienes no fueron realizados este procedimiento sobrevivieron. $(P=0.355)$

\section{Estadiaje}

La supervivencia cuando se diagnostica en el estadio I fue del $100 \%$, en estadio II de $96.5 \%$, en estadio III del 84\%, y en estadio IV es de 63\% ( $P=0.001)$ Vea la tabla 1. 
Tabla 1 Análisis de supervivencia de los pacientes diagnosticados de cáncer colorrectal en el hospital Eugenio Espejo durante el periodo 2010-2016 según el Estadio Clínico.

\begin{tabular}{lc}
\hline Estadiaje de cáncer de colon & Supervivencia a los 6 años \\
\hline I & $100 \%$ \\
II & $96.5 \%$ \\
III & $84 \%$ \\
IV & $63 \%$ \\
\hline
\end{tabular}

\section{Comorbilidad existente y estadiaje}

Los pacientes que presentaban comorbilidades en el momento del diagnóstico de cáncer de colon tuvieron una supervivencia de $100 \%$ si se encontraban en el estadio I y II, mientras en el estadio III la supervivencia fue del $85 \%$, el diagnóstico en estadio IV tuvo una mortalidad a 6 años del $100 \%(P=0.007)$.

\section{Supervivencia según el diagnóstico de los pacientes}

La sobrevida a los 6 años de tratamiento de los pacientes con diagnóstico de cáncer de colon y recto fue del $57 \%$. La supervivencia en cáncer rectal es del 39\%, cáncer de colon ascendente y ciego el $79 \%$, cáncer de colon descendente y sigma es del $83 \%$, en cáncer de colon transverso y sigma es del $100 \%$ en el periodo estudiado $(P=0.887)$. Tabla 2 .

Tabla 2 Localización del cáncer de colon en los pacientes atendidos en la Unidad de Oncología del Hospital Eugenio Espejo en el periodo 2010 - 2016

\begin{tabular}{lcc}
\hline Diagnóstico & Frecuencia $\mathbf{n = 3 9 5}$ & $\%$ \\
\hline Cáncer de colon y recto & 159 & 40.3 \\
Cáncer rectal & 113 & 28.6 \\
Cáncer de colon ascendente y ciego & 60 & 15.2 \\
Cáncer de colon descendente y sigma & 38 & 9.6 \\
Cáncer de colon transverso & 18 & 4.6 \\
Cáncer de colon sigmoideo & 7 & 1.8 \\
\hline
\end{tabular}

\section{Discusión}

En Ecuador la tasa de mortalidad por cáncer es de 4.9 por 100 mil habitantes (INEC-2016). En Estados Unidos es la tercera causa de muerte en hombres y mujeres [4, 6] y parece estar condicionado a factores medio-ambientales ya que el porcentaje de casos asociados con un patrón heredo familiar es solamente del $5 \%$. Por esta razón es de suma importancia tomar en cuenta todos los factores que están anclados al mismo. Existe un consenso acerca de la relación entre el consumo de carne roja y procesada y la obesidad (especialmente en hombres) lo que aumenta el riesgo de padecer CCR [7-9]. Aunque la mortalidad global se redujo gracias a las mejoras en el diagnóstico y tratamiento precoz, su incidencia se incrementa, pues de cada 100 casos de cáncer que se presentan 6 son de colon, con un crecimiento de $50 \%$ de los casos de esta enfermedad en los últimos 30 años [10]. Probablemente, esto se relacione con el progresivo envejecimiento de la población y a los cambios en el estilo de vida hacia un patrón más occidental [10].

Un estudio realizado por el Hospital Universitario Ramón y Cajal (Madrid, España) evidenció que los programas de detección precoz mediante la implantación de un cribado en poblaciones de riesgo medio demostró ser costo-eficaz, con un descenso de la mortalidad por CCR 
entre el 30-70\%. En España, cada Comunidad Autónoma ha desarrollado su propio programa y ha sido el País Vasco la primera en comunicar resultados positivos [11].

La literatura médica indica que este cáncer es más prevalente en hombres que en mujeres [12], así, en un análisis ecológico de series de tiempo para estudiar la incidencia, mortalidad y supervivencia del cáncer colorrectal en Cali (Colombia) registrados entre 1962-2012, la tasa de incidencia aumentó más en mujeres que en hombres, mientras que la tasa de mortalidad sólo se incrementó en los hombres en todos los grupos de edad [13]. A pesar de ello, hay que tomar en cuenta que la supervivencia relativa a cinco años fue independiente del sexo y aumentó a $29.7 \%$ en el periodo $1995-1999$ y a 39.8\% durante el periodo 2000-2004 [13], sin embargo, en el presente estudio hay un predominio de mujeres (62.3\%) sin que se encuentren diferencias significativas.

El diagnóstico muchas veces se realiza tardíamente ya que las manifestaciones clínicas pueden ser inespecíficas en relación a su localización y en la mayoría de casos es asintomática [14]; y, si se presenta en personas de baja escolaridad o en presencia de comorbilidades, como lo observado en el presente estudio; lo cual, se asocia frecuentemente a pobres condiciones de vida, la posibilidad de un diagnóstico oportuno se reduce aún más. En las campañas hospitalarias una de las principales pruebas que se realiza como sistema de detección oportuna de cáncer de colon, es la prueba de guayaco o sangre oculta en heces; sin embargo, en este estudio se observó que tan solo al 14.5\% pacientes se realizó este examen y en el 5\% de ellos el resultado fue positivo [15], de la misma manera exámenes especializados de imagen son utilizados usualmente para confirmar una sospecha diagnóstica, Hay que señalar que el pronóstico del cáncer de colon depende del grado de penetración en la pared intestinal y de la presencia de metástasis en ganglios regionales y a distancia. No existen estudios aleatorios de control para evaluar si la colonoscopia aislada de escrutinio reduce la incidencia o la mortalidad por cáncer colorrectal en personas con riesgo; sin embargo, dos grandes estudios de cohorte, el Estudio Multicéntrico Italiano y el Estudio Nacional de Pólipo revelan una reducción en la incidencia de cáncer colorrectal en comparación con los grupos control [15, 16]. La lesión premaligna del cáncer colorrectal es el pólipo adenomatoso y displasia plana, siendo el tipo histológico más común el carcinoma y subtipo adenocarcinoma (Asociación Española Contra el Cáncer, 2014). El tratamiento depende del estadiaje, es por esto que es importante hacer una detección oportuna [17], además, están las pruebas de diagnóstico con marcadores moleculares y genéticos que son útiles en el tratamiento y seguimiento de estos pacientes [18]. Lamentablemente todos estos medios diagnósticos dependen de la disponibilidad de recursos asignados a los hospitales públicos como el Hospital Eugenio Espejo en que se realizó el presente estudio por parte del Estado, sin que existan políticas sanitarias nacionales de promoción de salud y prevención para evitar este tipo de cáncer.

Por otra parte, la supervivencia de este tipo de cáncer depende del estadio en el que se encuentre en el momento del diagnóstico, observándose que el estadio I es de mejor sobrevida (100\%) a 6 años y va decreciendo en cada estadio llegando a 63\% en el estadio IV, lo que refuerza lo ya antes anotado, es decir, la necesidad de acciones de promoción de la salud y prevención [17, 19].

Actualmente en México, más del $80 \%$ de los pacientes presentan estadios avanzados al momento de su diagnóstico (estadios III y IV). La tasa de supervivencia relativa a 5 años para todos los cánceres diagnosticados entre el 2004 y 2010 fue del 68\% y aumenta hasta el 90\% cuando son detectados en estadios tempranos [15]. En países como Estados Unidos, se enfatiza también la importancia de la detección temprana, los rangos de supervivencia a cinco 
Nota del Editor La Revista Oncología Ecu permanece neutral con respecto a los reclamos jurisdiccionales en mapas publicados y afiliaciones institucionales. años son significativamente diferentes por estadio, siendo del $90 \%$ para enfermedad localizada, evidentemente ligada a la detección temprana $[15,17]$.

No se encontró asociación en el presente estudio con otros factores descritos en la literatura médica, como lo observado en el estudio realizado por la Universidad de Barcelona (España) que establece como factores protectores a la dieta, sobre todo el consumo de pescado y la ingesta de fibra [19]. También describe como factores de riesgo de padecer cáncer de colon al consumo de alcohol (más de $30 \mathrm{~g}$ diarios) y, específicamente, el consumo de cerveza y vino, al igual que el consumo de tabaco [20-21]. Además, se han descrito la acción de la vitamina D como protector del cáncer de colon [22], se requieren estudios prospectivos de y de cohorte para establecer estos factores de riesgo.

\section{Conclusiones}

La supervivencia en el presente estudio se asoció al nivel de instrucción de los pacientes, al estadiaje del cáncer, y al diagnóstico temprano sobre todo en pacientes con comorbilidades. No se evidenció relación con factores de riesgo como tabaco, alcohol, presencia de pólipos, etc. Al ser menor la supervivencia en población sobre todo de baja escolaridad, con comorbilidades, y al depender la supervivencia de un diagnóstico temprano, se hace necesario desarrollar políticas sanitarias nacionales que se objetiven en acciones integrales de prevención y educación de largo plazo, vigilando las variaciones de la tendencia del cáncer en general y el de colon en particular.

\section{Abreviaturas}

CCR: cáncer colorrectal.

\section{Información administrativa}

Archivos Adicionales

Ninguno declarado por los autores.

Agradecimientos

Los autores agradecen a todas las personas de las Instituciones que colaboraron en el desarrollo de esta investigación.

Contribuciones de los autores

Gabriela Ordóñez Ureta: conceptualización, validación, visualización, metodología, administración de proyecto, adquisición de fondos, investigación, escritura: revisión y edición.

Vinicio Navas Flores: conceptualización, curación de datos, análisis formal, investigación, recursos, software, redacción - borrador original.

Rene Buitrón Andrade conceptualización, análisis formal, adquisición de fondos, investigación, recursos, software. Todos los autores leyeron y aprobaron la versión final del manuscrito. 
Los autores no recibieron ningún tipo de reconocimiento económico por este trabajo de investigación. Los autores subsidiaron los gastos incurridos en el presente estudio.

Disponibilidad de datos y materiales

Existe la disponibilidad de datos bajo solicitud al autor de correspondencia. No se reportan otros materiales.

\section{Declaraciones}

Aprobación del comité de ética

No aplica a estudios observacionales con revisión de bases de datos o historias clínicas.

\section{Consentimiento para publicación}

No aplica para estudios que no publican imágenes explícitas como tomografías, resonancias, imágenes de exámenes físicos.

Conflictos de interés

Los autores declaran no tener ningún conflicto de competencia o interés.

Información de los autores

María Gabriela Ordóñez Ureta. Médico Residente Maternidad Nueva Aurora. Correo electrónico: gabbyta280587@gmail.com

Rafael Vinicio Navas Flores, Médico Tratante Servicio de Oncología Hospital Eugenio Espejo. Docente Facultad de Ciencias de la Salud Eugenio Espejo, Universidad UTE. Correo elctrónico: vnavas@ute.edu.ec ORCID: https://orcid.org/0000-0002-9838-3142

Rene Buitrón Andrade, PhD. Docente Pontificia Universidad Católica del Ecuador. Docente Facultad de Ciencias de la Salud Eugenio Espejo, Universidad UTE. Correo electrónico: renebuitrona@gmail.com

\section{Referencias}

1. Allemani C, Matsuda T, Di Carlo V, Harewood R, Matz M, Nikšić M, et al; CONCORD Working Group. Global surveillance of trends in cancer survival 2000-14 (CONCORD-3): analysis of individual records for 37513025 patients diagnosed with one of 18 cancers from 322 population-based registries in 71 countries. Lancet. 2018 Mar 17;391(10125):1023-1075. doi: 10.1016/S0140-6736(17)33326-3. Epub 2018 Jan 31. PMID: 29395269; PMCID: PMC5879496.

2. Salinas AM. Estudio retrospectivo de cáncer de apéndice en el Instituto Oncológico Nacional "Dr. Juan Tanca Marengo" SOLCA-Guayaquil 1991-2002. Rev. Oncol. Ecu. 2005;15(3-4):217-30. SU: roe-solca/333

3. Ganz PA, Casillas J, Hahn EE. Ensuring quality care for cancer survivors: implementing the survivorship care plan. Semin Oncol Nurs. 2008 Aug;24(3):208-17. doi: 10.1016/j.soncn.2008.05.009. PMID: 18687267.

4. Bray F, Ferlay J, Soerjomataram I, Siegel RL, Torre LA, Jemal A. Global cancer statistics 2018: GLOBOCAN estimates of incidence and mortality worldwide for 36 cancers in 185 countries. CA Cancer J Clin. 2018 Nov;68(6):394-424. doi: 10.3322/caac.21492. Epub 2018 Sep 12. Erratum in: CA Cancer J Clin. 2020 Jul;70(4):313. PMID: $\underline{30207593 .}$ 
5. Haraldsdottir S, Einarsdottir HM, Smaradottir A, Gunnlaugsson A, Halfdanarson TR. Krabbamein í ristli og endaparmi [Colorectal cancer - review]. Laeknabladid. 2014 Feb;100(2):75-82. Icelandic. doi: 10.17992/lbl.2014.02.531. PMID: 24639430.

6. Esposito K, Chiodini P, Capuano A, Bellastella G, Maiorino MI, Rafaniello C, Panagiotakos DB, Giugliano D. Colorectal cancer association with metabolic syndrome and its components: a systematic review with meta-analysis. Endocrine. 2013 Dec;44(3):634-47. doi: 10.1007/s12020-013-9939-5. Epub 2013 Apr 2. PMID: 23546613.

7. Van Blarigan EL, Fuchs CS, Niedzwiecki D, Zhang S, Saltz LB, Mayer RJ, et al. Association of Survival With Adherence to the American Cancer Society Nutrition and Physical Activity Guidelines for Cancer Survivors After Colon Cancer Diagnosis: The CALGB 89803/Alliance Trial. JAMA Oncol. 2018 Jun 1;4(6):783-790. doi: 10.1001/jamaoncol.2018.0126. Erratum in: JAMA Oncol. 2019 Apr 1;5(4):579. PMID: 29710284; PMCID: PMC6145685.

8. Vieira AR, Abar L, Chan DSM, Vingeliene S, Polemiti E, Stevens C, Greenwood D, Norat T. Foods and beverages and colorectal cancer risk: a systematic review and meta-analysis of cohort studies, an update of the evidence of the WCRF-AICR Continuous Update Project. Ann Oncol. 2017 Aug 1;28(8):1788-1802. doi: 10.1093/annonc/mdx171. PMID: 28407090.

9. Coleman MP, Quaresma M, Berrino F, Lutz JM, De Angelis R, Capocaccia R, et al.; CONCORD Working Group. Cancer survival in five continents: a worldwide population-based study (CONCORD). Lancet Oncol. 2008 Aug;9(8):730-56. doi: 10.1016/S1470-2045(08)70179-7. Epub 2008 Jul 17. PMID: 18639491.

10. Binefa G, Rodríguez-Moranta F, Teule A, Medina-Hayas M. Colorectal cancer: from prevention to personalized medicine. World J Gastroenterol. 2014 Jun 14;20(22):6786-808. doi 10.3748/wjg.v20.i22.6786. PMID: 24944469; PMCID: PMC4051918.

11. Simón MA.. Cribado del cáncer colorrectal. Rev. esp. enferm. dig. 2004;96(11):803-803. SU: $\underline{\text { scielo.es. }}$

12. Brenner H, Kloor M, Pox CP. Colorectal cancer. Lancet. 2014 Apr 26;383(9927):1490-1502. doi: 10.1016/S0140-6736(13)61649-9. Epub 2013 Nov 11. PMID: 24225001.

13. Cotés A, Clin P, Bravo L, Clin P, García L, Collazos P. Incidencia, mortalidad y supervivencia por cáncer colorrectal en Cali, Colombia, 1962-2012. Salud Pública Mex. 2014; 5(56), 457-464. SU: scielo.mx

14. Siegel RL, Miller KD, Goding Sauer A, Fedewa SA, Butterly LF, Anderson JC, Cercek A, Smith RA, Jemal A. Colorectal cancer statistics, 2020. CA Cancer J Clin. 2020 May;70(3):145-164. doi: 10.3322/caac.21601. Epub 2020 Mar 5. PMID: $\underline{32133645}$.

15. Wolf AMD, Fontham ETH, Church TR, Flowers CR, Guerra CE, LaMonte SJ, et al. Colorectal cancer screening for average-risk adults: 2018 guideline update from the American Cancer Society. CA Cancer J Clin. 2018 Jul;68(4):250-281. doi: 10.3322/caac.21457. Epub 2018 May 30. PMID: 29846947.

16. Kushi LH, Doyle C, McCullough M, Rock CL, Demark-Wahnefried W, Bandera EV, et al.; American Cancer Society 2010 Nutrition and Physical Activity Guidelines Advisory Committee. American Cancer Society Guidelines on nutrition and physical activity for cancer prevention: reducing the risk of cancer with healthy food choices and physical activity. CA Cancer J Clin. 2012 Jan-Feb;62(1):30-67. doi: 10.3322/caac.20140. PMID: 22237782.

17. Tarazona N, Gimeno-Valiente F, Gambardella V, Zuñiga S, Rentero-Garrido P, Huerta M, et al. Targeted nextgeneration sequencing of circulating-tumor DNA for tracking minimal residual disease in localized colon cancer. Ann Oncol. 2019 Nov 1;30(11):1804-1812. doi: 10.1093/annonc/mdz390. PMID: 31562764.

18. Sinicrope FA. Lynch Syndrome-Associated Colorectal Cancer. N Engl J Med. 2018 Aug 23;379(8):764-773. doi: 10.1056/NEJMcp1714533. PMID: $\underline{30134129}$. 
19. Magalhães $B$, Peleteiro $B$, Lunet $N$. Dietary patterns and colorectal cancer: systematic review and metaanalysis. Eur J Cancer Prev. 2012 Jan;21(1):15-23. doi: 10.1097/CEJ.0b013e3283472241. PMID: 21946864.

20. Klatsky AL, Li Y, Nicole Tran H, Baer D, Udaltsova N, Armstrong MA, Friedman GD. Alcohol intake, beverage choice, and cancer: a cohort study in a large kaiser permanente population. Perm J. 2015 Spring;19(2):2834. doi: 10.7812/TPP/14-189. Epub 2015 Mar 1. PMID: 25785639; PMCID: PMC4403578.

21. Cheng J, Chen Y, Wang X, Wang J, Yan Z, Gong G, et al. Meta-analysis of prospective cohort studies of cigarette smoking and the incidence of colon and rectal cancers. Eur J Cancer Prev. 2015 Jan;24(1):6-15. doi: 10.1097/CEJ.0000000000000011. PMID: 24722538.

22. Feldman D, Krishnan AV, Swami S, Giovannucci E, Feldman BJ. The role of vitamin D in reducing cancer risk and progression. Nat Rev Cancer. 2014 May;14(5):342-57. doi: 10.1038/nrc3691. Epub 2014 Apr 4. PMID: 24705652.

DOI: Digital Object Identifier PMID: PubMeD Identifier SU: Short URL 